\title{
Aprendizaje del protocolo de la valoración a través del marco de la enseñanza para la comprensión
}

\author{
Learning the assessment protocol through the \\ framework of teaching for understanding
}

José Eduardo Cifuentes-Garzón'

Recibido: mayo 06 de 2020 Aceptado: agosto 31 de 2020

\section{Resumen}

Este artículo, reporta los hallazgos de un estudio cuyo objetivo fue analizar la incidencia de una unidad didáctica diseñada en el marco de la Enseñanza para la Comprensión (EpC), en el aprendizaje del protocolo de la valoración en profesores de educación básica secundaria. Se optó por una investigación de enfoque cualitativo, con el diseño de investigación acción pedagógica, adelantada con la participación de 10 profesores de una institución educativa pública colombiana. Se utilizaron como instrumentos: la rutina de pensamiento "antes pensaba... ahora pienso", el taller investigativo y el protocolo de la valoración. Los resultados muestran que, con la implementación de los momentos de la exploración del tópico, la investigación guiada y el proyecto final de síntesis, los profesores aprendieron y aplicaron el protocolo de la valoración. Se concluye que la transferencia a las prácticas de aula de la EpC y el protocolo de la valoración, son oportunidades para el mejoramiento de los procesos de evaluación de los aprendizajes en estudiantes, a la vez que permiten refinar los procesos de planeación de clases en los profesores.

Palabras clave: aprendizaje, protocolo de la valoración, profesores, educación básica secundaria.

\begin{abstract}
This article reports the findings of a study whose objective was to analyze the incidence of a didactic unit designed in the framework of Teaching for Comprehension (Teaching for Comprehension (Teaching for Comprehension), in the learning of the assessment protocol in teachers of basic secondary education. A qualitative approach research was chosen, with the pedagogical action research design, carried out with the participation of 10 teachers from a Colombian public educational institution. The instruments used were: the thinking routine "before I thought ... now I think", the research workshop and the assessment protocol. The results show that, with the implementation of the moments of the exploration of the topic, the guided research and the final synthesis project, the teachers learned and applied the assessment protocol. It is concluded that the transfer to the classroom practices of the $\mathrm{EpC}$ and the evaluation protocol are opportunities for the improvement of the processes of evaluation of the learning in students, at the same time that they allow to refine the processes of planning of classes in the teachers.
\end{abstract}

Keywords: learning, assessment protocol, teachers, secondary education.

1 Licenciado en Educación Básica con Énfasis en Matemáticas, Humanidades y Lengua Castellana. Doctor en Educación y Sociedad, Secretaría de Educación de Cundinamarca, Colombia. E-mail: josecifuentes1980@gmail.com

ORCID: https://orcid.org/0000-0002-5602-957X 


\section{Introducción}

En la actualidad, "La sociedad del conocimiento y el aprendizaje a lo largo de la vida suponen nuevos retos a la educación y a la investigación educativa, la cual ha mostrado un incremento importante en las últimas décadas" (Mosquera, 2019). Tal es el caso de las prácticas de aula, las cuales requieren del interés de los profesores por perfeccionar los procesos de planeación y desarrollo de las clases, repensar las estrategias didácticas y las maneras de evaluar los aprendizajes. En esta perspectiva, Buitrago (2018), considera que "en muchas ocasiones los contenidos siguen siendo rígidos, las metodologías repetitivas, las perspectivas reducidas, el desarrollo de habilidades cuestionables y el disfrute del aprendizaje ocasional".

En consecuencia, se debe potenciar "el trabajo con los profesores sobre todos aquellos indicadores que caracterizan la buena enseñanza, en un marco de trabajo reflexivo y colaborativo, que contribuya al crecimiento profesional y a una mayor comprensión de la práctica de enseñanza" (Ravanal, 2019). En este contexto, surge el estudio titulado: Trayectorias de pensamiento en profesores sobre el protocolo de la valoración con la implementación del marco de la enseñanza para la comprensión, producto de las reflexiones de los profesores acerca de la manera como están valorando el trabajo de los estudiantes y la forma como realizan los procesos de realimentación de los aprendizajes.

La investigación se orientó por la pregunta: ¿Cómo la implementación de una unidad didáctica basada en el marco de la enseñanza para la comprensión, incide en el aprendizaje del protocolo de la valoración en profesores de educación básica secundaria?. En este sentido, se formuló como objetivo general: analizar la incidencia de una unidad didáctica diseñada en el marco de la enseñanza para la comprensión, en el aprendizaje del protocolo de la valoración en profesores de educación básica secundaria, de la Institución Educativa Departamental Misael Gómez, de Villagómez, Cundinamarca, Colombia.
Para cumplir con el objetivo general, se establecieron las siguientes acciones: i) describir los saberes previos que tienen los docentes sobre el protocolo de la valoración o escalera de la realimentación; ii) diseñar e implementar una unidad didáctica basada en el marco de la enseñanza para la comprensión, a fin de fomentar el aprendizaje del protocolo de la valoración en profesores de educación básica secundaria; y iii) valorar el impacto de la unidad implementada desde el marco de la enseñanza para la comprensión en el aprendizaje del protocolo de la valoración, en profesores de educación básica secundaria.

\section{Marco teórico y metodología}

\subsection{Fundamento teórico}

En lo teórico, se hizo un abordaje sobre: el protocolo de la valoración, la realimentación, el aprendizaje en profesores y la enseñanza para la comprensión, conceptos que se describen a continuación.

En cuanto al protocolo o escalera de la valoración. Es una herramienta para ofrecer y recibir realimentación. Se recomienda tener en cuenta en la conversación, los siguientes pasos: aclarar (a través de preguntas encaminadas a entender mejor algunos puntos o conocer ideas que no se han expresado); valorar (hacer énfasis en los puntos positivos, en las fortalezas, en aspectos interesantes y en los comentarios honestos del trabajo de la otra persona); expresar inquietudes (preocupaciones, dificultades o desacuerdos con algunas de las ideas en cuestión); y hacer sugerencias (dar recomendaciones) (Wilson, 2002).

Respecto a la realimentación. “Es un componente esencial en el proceso de enseñanza y aprendizaje, que provee al estudiante de reflexión sobre sus actos y consecuencias, le permite llegar a las metas y objetivos" (Vives \& Varela, 2013). Es decir, "es un proceso dialógico, pedagógico y fundamental en los procesos evaluativos de los diferentes cursos" (Sánchez \& Puertas, 2011). Se identifican "cuatro tipos de retroalimentación 
(Hattie \& Timperley, 2007): centrada en la tarea, en el proceso de la tarea, en la autorregulación y en la propia persona, a los que sumamos un quinto, la retroalimentación centrada en el contenido" (Canabal \& Margalef, 2017). La realimentación, "Si se ofrece en el momento oportuno, cuando el estudiante está actuando en un contexto determinado, es una herramienta poderosa para el cambio" (Vives \& Varela, 2013) y promueve las comprensiones profundas de los aprendizajes.

En relación con el aprendizaje en profesores. Para Cifuentes (2018), "estudiar las maneras como aprenden los profesores permite comprender la movilización de sus saberes y proyectar la transferencia a las prácticas de aula". Dichos aprendizajes, "se deben a la interacción con los saberes previos, el diálogo pedagógico entre pares, el contacto con otras fuentes del conocimiento y el desarrollo de la práctica pedagógica" (Cifuentes, 2019). En este sentido, resulta pertinente seguir indagando sobre cómo los profesores aprenden, cómo son las trayectorias que siguen para generar comprensiones. Según Nicastro y Greco, (2012), "Al hablar de trayectoria nos referimos a un recorrido, un camino en construcción permanente (...) que implica a sujetos en situación de acompañamiento". De acuerdo con Ghitis (2012), las trayectorias del pensamiento son entendidas como "los diferentes recorridos realizados por las personas para construir un concepto, con la vinculación de los aspectos teóricos y las experiencias vividas".

En lo referente al marco de la enseñanza para la comprensión. Se concibe como "una visión de la educación que pone la comprensión, ante todo. Esta forma de concebir la educación nos invita a reflexionar sobre nuestro trabajo en el aula y en la institución de una manera diferente" (Barrera \& León, 2014). De acuerdo con Stone (1999), este marco se centra en cuatro elementos: Ios tópicos generativos (son temas significativos que vale la pena comprender); las metas de comprensión (aquello que los estudiantes deben comprender, en términos de: conocimiento, método, propósito y comunicación); los desempeños de comprensión (son las acciones para motivar el aprendizaje de los alumnos, con la aplicación y ampliación de los saberes, a partir de: la exploración del tópico, investigación guiada y proyecto final de síntesis); y la valoración continua (permite controlar el avance de los estudiantes, por medio de evaluaciones diagnósticas continuas de sus desempeños).

\subsection{Métodos}

La investigación se enmarcó en el enfoque cualitativo, en la medida en que identifica a "los sujetos de investigación en su singularidad... se inserta en la búsqueda de significados atribuidos por los sujetos a sus experiencias sociales, parte de la realidad de los mismos y a ellos retorna de forma crítica y creativa" (Belén, 2019). De acuerdo con Cerrón (2019), la investigación cualitativa aplicada en educación, permite la generación de propuestas para el mejoramiento de la estructura de la realidad social emergente de la formación de estudiantes, los docentes y la comunidad, aportando a la producción, transferencia y renovación del saber pedagógico.

La investigación es de alcance descriptivo. Este tipo de estudio busca "especificar propiedades y características importantes de cualquier fenómeno que se analice. Describe tendencias de un grupo o población" (Hernández, Fernández \& Baptista, 2014). El diseño utilizado fue el de la investigación acción pedagógica. Esta es "una modalidad de investigación social que busca explorar las intimidades de las prácticas pedagógicas, en el ámbito de la escuela..." (Ávila, 2005).

En esta perspectiva, "La práctica empírica o real de la educación obliga a transformar la práctica teórica, labor que compete a los practicantes de la educación: los maestros" (Restrepo, 2006). Por tal razón, "En este proceso de reflexión y transformación continua de la práctica para hacer de ella una actividad profesional guiada por saber pedagógico apropiado... la investigación acción pedagógica, se ofrece como escenario y método potenciador" (Restrepo, 2006). En efecto, se optó por este tipo de investigación, a fin de comprender las trayectorias del pensamiento 
de los profesores, frente al aprendizaje del protocolo de la valoración, como oportunidad para la implementación de estrategias que permitan fortalecer el desarrollo de la evaluación formativa. Para ello, se utilizaron como instrumentos de recolección de la información:

La rutina de pensamiento "antes pensaba... ahora pienso": las rutinas de pensamiento, son "instrumentos que ayudan a generar acciones concretas de pensamiento, ayudando en la comprensión de los contenidos, y desarrollando las capacidades de: interpretar, argumentar y proponer" (Cifuentes, 2015). Una de estas rutinas es "antes pensaba... ahora pienso", la cual consiste en: indagar las ideas previas sobre el tema objeto de estudio, luego intervenir con diferentes acciones pedagógicas y finalmente averiguar las nuevas comprensiones, después de haber abordado la temática con diferentes desempeños. "Esta rutina ayuda a los estudiantes a reflexionar sobre su pensamiento acerca de un tema o cuestión y a explorar cómo y por qué el pensamiento ha cambiado" (Richarth, Church \& Morrison, 2014). Es decir, ayuda a "reflexionar sobre su propio pensamiento a cerca de un tema e identificar cómo sus ideas se han desarrollado a lo largo del tiempo (Richarth et al., 2014).

El taller pedagógico investigativo: es una herramienta académica mediante la cual los docentes comparten experiencias, intercambian conocimientos y llevan a la práctica acciones educativas para el mejoramiento del trabajo en las prácticas de aula (Alfaro \& Badilla, 2017). En el presente estudio, el diseño del taller pedagógico investigativo, se fundamentó en la secuencia de una unidad didáctica en el marco de la enseñanza para la comprensión.

El protocolo de la valoración: esta escalera consta de cuatro peldaños: formular preguntas aclaratorias, valorar, expresar inquietudes y dar sugerencias o recomendaciones. Es efectiva para proporcionar retroalimentación específica, constructiva, respetuosa, orientada, honesta, oportuna, diferenciada y amigable (Libedinsky, 2016).

En la investigación participaron 10 profesores de educación básica secundaria y media técni- ca, de la Institución Educativa Departamental Misael Gómez, del municipio de Villagómez, Cundinamarca. Fue pertinente desarrollar el estudio en esta institución por la facilidad de acceso a los profesores participantes, ya que el investigador se desempeñaba como directivo docente de la institución educativa, único establecimiento prestador del servicio educativo en este municipio.

Durante el desarrollo del estudio, se adelantaron tres fases: la primera fue el diagnóstico de saberes previos, con la primera parte de la rutina de pensamiento "antes pensaba... ahora pienso"; la segunda corresponde a la intervención, a través de una unidad didáctica diseñada en el marco de la enseñanza para la comprensión; y la tercera concierne a la valoración de la efectividad de la intervención, a través de la segunda parte de la rutina "antes pensaba... ahora pienso" y la implementación del protocolo de la escalera de la valoración, como se relaciona en los siguientes apartados.

\section{Resultados y discusión}

A continuación, se exhiben los hallazgos desde los momentos desarrollados en el proceso de la investigación: exploración del tópico, con la primera parte de la rutina de pensamiento "antes pensaba... ahora pienso"; investigación guiada desde la aproximación a los fundamentos teóricos; y proyecto final de síntesis, con la segunda parte de la rutina de pensamiento "antes pensaba... ahora pienso" y la aplicación de un protocolo de la valoración. El investigador realizó la sistematización de la información, siendo fiel a las voces de los participantes y luego da sus interpretaciones a la luz de la teoría y la praxis, en la sección de interpretación de los resultados:

\subsection{Exploración del tópico}

Primera parte de la rutina de pensamiento "antes pensaba... ahora pienso": los profesores escribieron las ideas previas que tenían sobre la escalera de la valoración, como se presenta en la tabla 1. 
Tabla 1. Primer momento de la rutina "antes pensaba... ahora pienso".

\begin{tabular}{|c|c|}
\hline Instrucción & Aspectos clave \\
\hline \multirow{8}{*}{$\begin{array}{l}\text { Escribir } \\
\text { ¿Qué se le viene } \\
\text { a la mente } \\
\text { cuando escucha } \\
\text { la expresión } \\
\text { “Protocolo } \\
\text { de la valoración"? }\end{array}$} & $\begin{array}{l}\text { Profesor } 1 \text { (P1). Es una gradación de niveles sobre cómo se valora el trabajo de los } \\
\text { educandos en el proceso de enseñanza y aprendizaje teniendo en cuenta su esfuerzo, } \\
\text { valores y proceso, entonces se plantea una evaluación integral para permitir que se } \\
\text { construya una enseñanza y aprendizaje más efectivo en el sentido de que se dialoga } \\
\text { sobre lo visto y se construyen nuevos conocimientos. }\end{array}$ \\
\hline & $\begin{array}{l}\text { P2. Son valores con ciertos criterios establecidos. Cada nivel tiene un desempeño que } \\
\text { se debe cumplir; en el caso que no se cumplan se realizan actividades para mejorar e } \\
\text { ir superando y se irá escalonando hasta llegar a lo máximo. }\end{array}$ \\
\hline & $\begin{array}{l}\text { P3. Es un proceso que cataloga a alguien en qué nivel de aprendizaje se encuentra, } \\
\text { después de recibir cierta información sobre un tema, la cual puede ascender de acuerdo } \\
\text { con sus capacidades intelectuales, a su dedicación, atención, responsabilidad y ante } \\
\text { su actitud. Esta escala se le da criterios o parámetros para evaluar y de acuerdo con } \\
\text { los resultados se le asigna una letra y/o numeración que identifique su nivel al cual } \\
\text { alcanzó o logró obtener. }\end{array}$ \\
\hline & $\begin{array}{l}\text { P4. Es la forma como un docente evalúa a un estudiante, teniendo en cuenta el sistema } \\
\text { de valoración cuantitativa el cual radica en la siguiente escala } 1.0 \text { (Muy bajo)- } 5.0 \\
\text { (Superior). En Colombia se ha estandarizado distintos modelos de valoración como es } \\
\text { el caso cualitativo, en donde se valora lo procedimental, actitudinal y cognitivo. }\end{array}$ \\
\hline & $\begin{array}{l}\text { P5. Pienso en unos criterios para tener en cuenta para valorar una acción o una actividad } \\
\text { de una persona o grupo de personas. }\end{array}$ \\
\hline & $\begin{array}{l}\text { P6. Concibo un camino, un proceso con unas tareas, estándares y propósitos } \\
\text { permanentes que se van alcanzando a medida que voy escalando cada uno de los } \\
\text { peldaños que tiene la Escalera de la vida en el ámbito personal, familiar, profesional y } \\
\text { laboral, con una connotación socioeconómica, ética y religiosa. }\end{array}$ \\
\hline & $\begin{array}{l}\text { P7. Por "Escalera de la valoración" entiendo un proceso que se da para realizar la } \\
\text { valoración de un trabajo a través de múltiples personas o procesos que permitan tener } \\
\text { una secuencia de menor a mayor por medio de la cual se complejiza dicha valoración, } \\
\text { además puede ser una herramienta de constante "valoración" del trabajo realizado } \\
\text { en el Colegio. }\end{array}$ \\
\hline & $\begin{array}{l}\text { P8. Es un proceso que permite medir aprendizajes, conocimientos, avances de la } \\
\text { formación y evolución de los estudiantes, es donde se emiten opiniones frente a } \\
\text { parámetros que están establecidos en la evaluación; además debe ser una herramienta } \\
\text { que le permite al docente reflexionar, para cambiar y mejorar la práctica. }\end{array}$ \\
\hline
\end{tabular}

En la exploración del tópico, se evidenciaron los saberes previos del grupo de profesores participantes frente al protocolo de la valoración, los cuales se pueden agrupar en cuatro aspectos: i) como parámetros, criterios, gradación en niveles y valores para evaluar los aprendizajes cuantitativamente; ii) como un proceso de evaluación integral, con tareas para valorar un trabajo en los aspectos: procedimental, actitudinal y cognitivo por parte de varias personas; iii) como peldaños para determinar los avances de los aprendizajes en las diferentes dimensiones del desarrollo humano (personal, familiar, profesional y laboral); y iv) como una herramienta que permite al docente reflexionar, transformar y mejorar la práctica pedagógica.

Llama la atención el último aspecto como conciben los profesores inicialmente el protocolo de la valoración, al referirse a la práctica pedagógica reflexiva, entendida como "el proceso sistemático del docente, para: cuestionar sus creencias, 
pensamientos y acciones pedagógicas con el propósito de resolver un problema pedagógico y mejorar sus propias prácticas, que resulten luego en la mejora de los aprendizajes de los estudiantes" (Vega \& Appelgren, 2019). Esto significa que han tenido aproximaciones a la investigación de procesos de aula o tienen la intención de hacerlo, lo cual responde a las exigencias educativas actuales, puesto que "Se requiere un docente capaz de problematizar, de indagar, de formular hipótesis, de buscar soluciones a sus problemas educativos cotidianos" (Romero \& Sanz, 2017).

\subsection{Investigación guiada}

Intervención a partir de una unidad didáctica en el marco de la enseñanza para la comprensión: después de conocer los saberes previos de los profesores, se construyó una unidad en el marco de la enseñanza para la comprensión sobre el protocolo de la valoración, bajo el tópi- co generativo: "Si las prácticas de aula quieres mejorar... la escalera de la valoración puedes implementar", la cual se desarrolló en 3 sesiones, de 3 horas cada una. Las metas de comprensión, se formularon desde las diferentes dimensiones, en los siguientes términos:

Los profesores comprenderán: la definición y la estructura de la escalera de la valoración (conocimiento); cómo desarrollar el protocolo de la escalera de la valoración en la realimentación de los preparadores de clase (método); la importancia de la escalera de la valoración en los procesos de mejoramiento de las prácticas de aula (propósito); y la necesidad de compartir con otros colegas lo aprendido sobre el protocolo de la escalera de la valoración, a través de socializaciones de rutinas de pensamiento y protocolos implementados (comunicación). Para lograr lo anterior, se planearon los desempeños de comprensión relacionados en la tabla 2:

Tabla 2. Desempeños de comprensión sobre el protocolo de la valoración.

\begin{tabular}{|c|c|c|}
\hline Exploración del tópico & Investigación guiada & $\begin{array}{l}\text { Proyecto final } \\
\text { de síntesis }\end{array}$ \\
\hline $\begin{array}{l}\text { 1. Rutina de pensamiento "antes } \\
\text { pensaba... ahora pienso": Escribir } \\
\text { aquello que se le viene a la mente } \\
\text { cuando escucha la expresión "Es- } \\
\text { calera de la valoración". Sin haber } \\
\text { leído nada al respecto. Son sus } \\
\text { ideas previas. } \\
\text { Socializar con el compañero más } \\
\text { cercano las respuestas. } \\
\text { 2. Rutina de pensamiento "cír- } \\
\text { culos de puntos de vista" para } \\
\text { conocer los saberes previos sobre } \\
\text { el modelo de evaluación emplea- } \\
\text { do en las sesiones pedagógicas. } \\
\text { Enumerar el grupo de } 1 \text { a } 3 \text {. Los } 1 \\
\text { representan la maestra tradicio- } \\
\text { nal, los } 2 \text { el niño y los } 3 \text { la maestra } \\
\text { innovadora. }\end{array}$ & $\begin{array}{l}\text { 3. Leer el documento “La Retroalimentación a través de } \\
\text { la Pirámide”. Por Daniel Wilson. Traducido al español } \\
\text { por Patricia León Agustí, Constanza Hazelwood y María } \\
\text { Ximena Barrera. } \\
\text { 4. Rutina de pensamiento OFP. Escribir una oración, una } \\
\text { frase y una palabra que le haya llamado poderosamente } \\
\text { la atención de la lectura del punto anterior. } \\
\text { 5. Organizador gráfico. Con las respuestas de la rutina } \\
\text { OFP, elaborar un organizador gráfico que demuestre } \\
\text { sus comprensiones (mapa mental, mapa conceptual, } \\
\text { red de ideas, entre otros). } \\
\text { 6. Observar el vídeo: https://www.youtube.com/ } \\
\text { watch?v=T3tKmhISowM } \\
\text { Desarrollar la rutina de pensamiento “Ver-Pensar- } \\
\text { Conectar": ¿Qué vio en el vídeo?, ¿Qué pensó sobre lo } \\
\text { que vio? y ¿Con qué situaciones de su quehacer docente } \\
\text { conectó lo visto en el vídeo? }\end{array}$ & $\begin{array}{l}\text { 7. Rutina de pensamiento } \\
\text { "antes pensaba... ahora } \\
\text { pienso": Escribir aquello } \\
\text { que ahora piensa de "La } \\
\text { escalera de la valoración". } \\
\text { Ambas Rutinas de pensa- } \\
\text { miento "antes pensaba... } \\
\text { ahora pienso" y "OFP", se } \\
\text { socializarán en reunión de } \\
\text { profesores. } \\
\text { 8. Realimentación de pre- } \\
\text { paradores de clase: Cada } \\
\text { docente diseña una sesión } \\
\text { de clase y la socializa con } \\
\text { los colegas, aplicando el } \\
\text { protocolo de la escalera } \\
\text { de la valoración. }\end{array}$ \\
\hline \multicolumn{3}{|l|}{ Valoración continua } \\
\hline $\begin{array}{l}\text { Socialización y realimentación a } \\
\text { las respuestas de la rutina imple- } \\
\text { mentada. }\end{array}$ & $\begin{array}{l}\text { Se socializan las respuestas de la rutina implementada, } \\
\text { las cuales se realimentan con los aportes de todos. }\end{array}$ & $\begin{array}{l}\text { Realimentación de cada } \\
\text { unidad por parte de los } \\
\text { demás colegas. }\end{array}$ \\
\hline
\end{tabular}


El anterior diseño de la unidad didáctica en el marco de la enseñanza para la comprensión, fue elaborado por el investigador y realimentado por cinco docentes con nivel de maestría, quienes laboran en el lugar donde se desarrolló el proyecto. La realimentación generó las siguientes transformaciones:

- En el tópico generativo, se cambió el verbo deber por poder. En las metas de comprensión, en la de conocimiento se agregó "definición y estructura de la escalera de la valoración".

- En la de comunicación se agregó "a través de socializaciones de rutinas de pensamiento y protocolos implementados". En los desempeños de comprensión se agregaron los tiempos para cada desempeño.

- En la exploración del tópico, se agregó la socialización de una rutina de pensamiento y se agregó un desempeño, que consiste en una rutina de pensamiento: "Círculos de los puntos de vista".

- En la investigación guiada, se agregaron dos desempeños, el de elaborar un organizador mental y una rutina de pensamiento después de observar un video.

- En la valoración continua no se evidencian cambios.

De acuerdo con lo anterior, la unidad didáctica de intervención contó con la realimentación de expertos en el campo de la educación, que permitieron refinarla, a fin de conseguir mejores resultados en su implementación. Cuando se trabaja en colaboración, "La práctica pedagógica pasa de ser un ejercicio meramente rutinario a un ejercicio profesional, analítico y reflexivo, por medio de una organización clara y coherente en el diseño de unidades de comprensión, estructuradas bajo los parámetros de la EpC". (Villamizar, Montero, González \& Castillo, 2019).

Retomando la fase de la investigación guiada, los desempeños desarrollados en este momento, fueron: lecturas de documentos de autores expertos que han aportado a la consolidación del protocolo de la valoración, vídeos sobre el tema y el uso de rutinas de pensamiento (oración-frasepalabra OFP y ver-pensar-preguntarse), y organizadores gráficos. Estas fuentes permitieron que los profesores interpretaran, argumentaran y propusieran nuevos desempeños sobre el tópico generativo, combinando teoría y práctica. Al respecto, Villa (2019), señala:

(...) "la praxis investigativa como equilibrio teórico-práctico posibilita que los avances y hallazgos del proceso investigativo le sean útiles en principio a las personas que participan del proceso, como protagonistas de la investigación sobre su propia realidad" (p. 555).

\subsection{Proyecto final de síntesis}

Como se evidencia en el diseño de la unidad presentado en el apartado anterior, la sección del proyecto final de síntesis, se centra en completar la segunda parte de la rutina de pensamiento "antes pensaba... ahora pienso" y la aplicación del protocolo de la valoración a unos preparadores de clase de los docentes participantes en el estudio. A continuación, se relacionan los principales hallazgos de estos dos aspectos.

Nuevas respuestas al tema: después de haber desarrollado el taller sobre el protocolo de la valoración, los profesores transformaron y complementaron sus saberes, como se evidencia en la tabla 3. 
Tabla 3. Segundo momento de la rutina "Antes pensaba... ahora pienso".

\section{Instrucción}

Escribir

¿Qué piensa

ahora sobre el

Protocolo de

la valoración?

\section{Aspectos clave}

P1. Es una manera de pensar planteando la forma como se hará retroalimentación de los trabajos de los estudiantes tratando de construir un diálogo con los educandos y con sus saberes, no solamente moldeando lo que se quiere de ellos, también importante de hacer recomendaciones constructivas que vayan edificando su saber y criterio que los posibilite para extrapolar los conocimientos de los saberes escolares con la realidad de su entorno y del mundo actual.

P2. Es una herramienta que ayuda a reflexionar sobre la valoración de la comprensión con estos pasos: Clarificar. Al compartir un trabajo algunas ideas no pueden ser muy claras, lo que permite que a través de preguntas se pueda clarificar la información. Valorar. Después de recoger la información se da paso a la comprensión haciendo énfasis en aspectos positivos, fortalezas y aspectos interesantes del trabajo de los demás, generando confianza. Expresar inquietudes. También existen desacuerdos en algunas ideas, y es aquí donde se debe expresar las inquietudes y pensamientos honestos. Sugerir. Finalmente se hacen sugerencias para mejorar los trabajos y utilizarlos como retroalimentación positiva.

P3. Es aquella que permite emitir un juicio que valora saberes previos o saberes adquiridos durante un proceso de aprendizaje, donde los diversos agentes del proceso (Docente, estudiantes y compañeros), realizan una retroalimentación ya sea formal o informal y que a partir de ella refuerce su conocimiento, demostrando que el aprendizaje es un trabajo colaborativo. Esta escala permite que el estudiante en primera instancia realice su autoevaluación, luego aplique la coevaluación y heteroevaluación.

P4. Pienso que en la enseñanza el docente aplica durante todo el proceso todos los conceptos de valoración; tanto cualitativa como cuantitativa. En la cualitativa se ve reflejado en lo procedimental, actitudinal y cognitivo; es decir, en el ser, el saber y el saber hacer. De este modo también se han aplicado estrategias metodológicas al implementar las rutinas de pensamiento en el desarrollo de las clases y manejando un modelo para planear clases como es el caso de Tópico Generativo, las metas de Comprensión (conocimiento, método, propósito y comunicación) sujetos a los estándares; Desempeños de comprensión, el cual se ve reflejado en (la exploración del tópico, investigación guiada y proyecto final de síntesis).

P5. Herramienta que facilita tanto al docente como a los estudiantes reflexionar sobre las comprensiones de un tema o un concepto, permite al docente retroalimentar conceptos y procesos por medio de unos pasos sencillos como aclarar, valorar, hacer sugerencias y expresar inquietudes, además de ayudar a identificar los momentos en los cuales se requiere buscar metodologías diferentes para abordar los contenidos.

P6. La retroalimentación a través de la Pirámide facilita la labor docente, desde luego los procesos de enseñanza-aprendizaje implementando algunos componentes que sirven para planear, reflexionar, guiar las experiencias de valoración, crear múltiples maneras de valorar y retroalimentar para la comprensión. Desde esta perspectiva, la enseñanza se puede fortalecer de manera que los estudiantes tengan múltiples oportunidades para observar fenómenos, realizar experimentos, analizar datos, generar modelos, construir argumentos y comunicar o expresar sus ideas públicamente.

P7. Es un conjunto de herramientas novedosas y validas que permiten ayudar a cultivar una cultura de la valoración para la comprensión muy importante en nuestra labor docente, ya que permite que cada una de las actividades, estrategias de aprendizaje, y proyectos sean valorados de diferentes formas, con diferentes estilos, pero además de una manera más completa, los autores recomiendan que se tengan en cuenta los pasos: clarificar, valorar, expresar inquietudes y hacer sugerencias; estas herramientas hacen que el proceso de valoración se haga de manera ordenada, lo que permite además superar un poco la subjetividad que puede traer consigo el proceso evaluativo.

P8. Promueve la valoración informal, en la cual se pretende hacer una conversación con el estudiante para generar en él reflexión sobre su aprendizaje a fin de empezar a mejorar. Esta herramienta permite al docente guiar la construcción de experiencias de valoración, la retroalimentación debería ser utilizada por los maestros, estudiantes y personas en general, teniendo en cuenta que es importante clarificar lo que se está expresando, valorar, expresar inquietudes y hacer sugerencias, de esta manera se construye desde la reflexión el conocimiento. 
Respecto a los hallazgos de la segunda parte de la rutina "antes pensaba ahora pienso", se puede evidenciar la progresión en la comprensión del protocolo de la valoración de los profesores participantes en la investigación. Esto se manifiesta en la asociación el protocolo de la valoración con la realimentación que se debe dar a los estudiantes en los diferentes procesos evaluativos formativos, desde una perspectiva positiva. Lo conciben como una herramienta que ayuda a reflexionar sobre la valoración de la comprensión de temas y conceptos. Además, manifiestan un discurso teórico y práctico refinado en cuanto a los momentos del protocolo: aclarar, valorar, expresar inquietudes y sugerir.

Aplicación de protocolos de la valoración: en la tabla 4, se relacionan algunas expresiones dadas en la realimentación de la escalera de la valoración, sobre preparadores de clase socializados en reuniones de profesores.

Tabla 4. Ejemplos de realimentación del protocolo de la valoración.

\section{Momentos Aspectos clave de la realimentación}

Aclarar P1. ¿En qué consiste la investigación que deben hacer los estudiantes?, ¿Qué se obtiene de ahí?

P2. ¿En qué momento se da el tema a los estudiantes?, ¿Qué se hace con la investigación?, ¿Qué se hace con los mapas mentales?

P3. ¿Siempre planea en ese formato?, ¿Cuál puede ser un ejemplo de una ecuación lineal famosa?, ¿Cuál es la diferencia entre una ecuación lineal y una ecuación cuadráticas y cubicas?, ¿En cuánto tiempo en total se desarrollará la Unidad Didáctica?

P4. ¿Cómo resuelve las deficiencias que tienen los estudiantes respecto al tema?, ¿Cuál es el producto final esperado?, ¿Qué tiempo dispone para desarrollar la Unidad Didáctica?

Valorar P1. Interesante que lleva el conocimiento desde una mirada global hasta algo particular o local. Interesante utilizar material que está disponible en la institución para que los estudiantes hagan la investigación. P2. Me parecen interesantes la manera de investigar porque hace que los estudiantes sean autónomos en la búsqueda de la información. También me parece muy positivo que se usen los mapas mentales como estrategia metacognitiva y que la valoración de esos productos la realicen docente-estudiante. P3. El planeador de clase está muy bien organizado, define claramente los tiempos a utilizar y tiene mucha variedad en actividades didácticas lo que probablemente genera un excelente clima de aula. Además, es positiva la relación con el contexto local en la medida en que se invita a los estudiantes a relacionar el tema de las ecuaciones lineales con los negocios locales.

P4. Valoro positivamente que se haga una actividad para conocer los saberes previos de los estudiantes. Además, que el lenguaje utilizado es apropiado para familiarizar al estudiante con el tema.

Expresar P1. Propone mucho tiempo para desarrollar la rutina de pensamiento. Evidencio que no es viable el preocupaciones tiempo de investigación por que los estudiantes no lo aprovechan para realizar las actividades propuestas. P2. Me preocupa que algunas actividades no especifican las instrucciones que se seguirán.

P3. Me pregunto si es posible elaborar este tipo de material para todos los cursos en los que orienta clases al margen de los estudios de maestría, teniendo en cuenta la inversión de tiempo y recursos que requiere este tipo de unidad diseñada. Además, los tiempos que se demoran los estudiantes en realizar las actividades.

P4. Me preocupa la actitud de los estudiantes que no quieren trabajar, qué estrategias se pueden promover para mitigar las deficiencias en los estudiantes.

Sugerir P1.Desarrollar estrategias para la investigación, que se pueda evidenciar el producto. La socialización del producto final debería ser un ejercicio que se recoja al finalizar la clase para evidenciar el producto de esta. P2. Sugiero que se especifique en cada actividad qué se va a hacer con los productos realizados; de igual manera, mostrar los mapas mentales a todo el colegio, publicándolos en las carteleras de los pasillos. P3. Sugeriría la revisión de algunos de los tiempos sobre todo en la fase de proyecto final de síntesis.

P4. Las recetas matemáticas se pueden consolidar para tomarlas como modelo para aplicar en los diferentes contextos. Partir de un problema real para poder aplicar la fórmula o la receta respectiva. 
La implementación del protocolo de la valoración entre los profesores participantes en la investigación, para realimentar unas planeaciones de clase, facilitó el mejoramiento de la preparación de clase al recibir mutuamente sugerencias, en cuanto al conocimiento disciplinar y didáctico por docentes expertos en diferentes áreas del saber. Además, "la experiencia de compartir situaciones positivas y negativas, producto de la práctica docente, les permitió ganar confianza, junto con el desarrollo de un discurso en el marco de la cordialidad y respeto" (Aristizábal, 2019). En consecuencia, el aprendizaje entre colegas: "puede mejorar la enseñanza... las comunidades de aprendizaje profesional constituyen una estructura para el aprendizaje colaborativo, consistente en compartir las enseñanzas con homólogos... (Lo cual) requiere la autonomía de los docentes y una dedicación considerable en tiempo y recursos" (Unesco, 2017).

De acuerdo con lo expuesto anteriormente, hubo comprensiones profundas sobre el protocolo de la valoración por parte de los docentes participantes, producto de la implementación de la unidad didáctica diseñada en el marco de la enseñanza para la comprensión. Este marco, ha sido recreado y utilizado "en la formación docente en diversos países, convirtiéndose hoy en un interesante dispositivo que permite que educadores de diferentes niveles y regiones del mundo, reflexionen colaborativamente sobre la enseñanza" (Chaves \& Barrios, 2017)

La secuencia didáctica del marco de la enseñanza para la comprensión, a saber: exploración del tópico, investigación guiada y proyecto final de síntesis, permitió evidenciar el proceso de construcción del aprendizaje del protocolo de valoración o escalera de la realimentación, en un grupo de profesores de educación secundaria, de una institución de educación pública colombiana. Según Perkins (2014), los elementos de este marco, "representan una metodología para organizar el proceso de planificación y de conducción de la enseñanza, y constituyen una herramienta para poner en marcha al conocimiento, en el camino hacia la sabiduría".
Es decir, la secuencia misma de la enseñanza para la comprensión, facilitó en los profesores la comprensión del protocolo de la valoración, y a la vez permitió reflexionar sobre la manera como abordaban los procesos de enseñanza y de evaluación, en sus áreas de conocimiento. Con el desarrollo de estas unidades didácticas ocurren transformaciones en las prácticas de aula, debido a que "del hacer empírico el maestro pasa a un hacer reflexivo, a una práctica reflexiva que le permite remontar la rutina repetitiva para objetivar su práctica por escrito, con miras a continuar reflexionándola y transformándola en la misma acción" (Restrepo, 2006).

Estos ejercicios de capacitación y reflexión sobre temas propios de la labor docente, pueden "ayudar a los docentes a mejorar su trabajo cuando son conscientes de sus creencias, teorías, actitudes, valores... y, para ello, se les "obliga" a explicitarlas" (Bautista, 2017), situación ocurrida con el aprendizaje del protocolo de la valoración, que permitió entre otras cosas, reflexionar sobre las planeaciones de clase e implementarlo en los procesos evaluativos con los estudiantes.

\section{Conclusiones}

Es importante enunciar que el marco de la enseñanza para la comprensión y el protocolo de la valoración o escalera de la realimentación, pueden convertirse en oportunidades potentes para el mejoramiento de los procesos de evaluación de los aprendizajes en estudiantes y a la vez, refinar los procesos de planeación de clases en los profesores. Por esta razón, la investigación busca responder al interrogante ¿Cómo la implementación de una unidad didáctica basada en el marco de la enseñanza para la comprensión incide en el aprendizaje del protocolo de la valoración en profesores de educación básica secundaria?

En consecuencia, para determinar los saberes previos que tienen los docentes sobre el protocolo de la valoración o escalera de la realimentación, se analizaron las respuestas dadas en el primer momento de la rutina de pensamiento "antes pensaba... ahora pienso". En este punto, 
se observó que los profesores asociaron el protocolo de la valoración con procesos que han desarrollado en la institución y en las mismas prácticas de aula, lo cual se manifestó en las conexiones que lograron hacer, tales como: evaluación de aprendizajes cuantitativos en los estudiantes, la evaluación integral y formativa, la evaluación cualitativa para determinar los avances de los aprendizajes en las diferentes dimensiones del desarrollo, y como herramienta que potencia la transformación de las prácticas de aula.

Por otra parte, se logró construir una unidad didáctica en colaboración con un grupo de profesores, lo cual permitió tener un producto refinado que facilitó el proceso de enseñanza con los demás colegas participantes en la investigación. También se evidenció que la estructura misma del marco de la enseñanza para la comprensión, permite generar secuencias apropiadas que garantizan aprendizajes efectivos, toda vez que: parten de los saberes previos, se orientan los procesos investigativos para generar transformaciones en los saberes iniciales $y$, finalmente, se divulgan los aprendizajes con otros colegas.

Así mismo, mediante la evaluación del impacto generado por la unidad implementada desde el marco de la enseñanza para la comprensión en el aprendizaje del protocolo de la valoración, en profesores de educación básica secundaria, con la última parte de la rutina de pensamiento ahora pienso y con la implementación del protocolo de la valoración, se logró una mejora notoria en el discurso de los profesores. Esto se refleja en la medida en que verbalizaron acertadamente: la definición, importancia, utilidad y la manera de implementar el protocolo de la valoración con estudiantes, así como en el proceso de reflexión y mejoramiento de la planeación y ejecución de las prácticas de aula.

Por último, según las políticas públicas de los últimos gobiernos, Colombia se visiona para el 2025 como el país mejor educado de América Latina. Sin embargo, "aún enfrenta muchos retos, uno de los cuales es precisamente la formación docente y su importancia en la creación de am- bientes de aprendizaje significativos" (Castro, 2019). Para ello, "se requiere indagar cómo se promueve efectivamente el aprendizaje significativo y cómo se construye el conocimiento en las aulas, qué factores facilitan estos procesos y qué factores los dificultan" (Tovar, 2019). En este sentido, "investigar la práctica pedagógica en nuestro país sigue siendo un desafío que va más allá de las sistematizaciones, necesitamos conocer lo que ocurre desde la perspectiva de los múltiples actores que intervienen en el proceso" (Bolívar, 2019).

\section{Referencias}

Alfaro, A., \& Badilla, M. (2017). El taller pedagógico, una herramienta didáctica para abordar temas alusivos a la Educación Ciudadana. Revista Electrónica Perspectivas, 10, 81-146.

Aristizábal, A. (2019). Fortalecimiento de la identidad profesional docente en el ámbito personal del profesor. Tecné, Episteme y Didaxis, 46, 189-204.

Ávila, R. (2005). La producción de conocimiento en la investigación acción pedagógica (IAPE): balance de una experimentación. Educação e Pesquisa, São Paulo, 31 (3), 503-519.

Barrera, M., \& León, P. (2014). ¿De qué manera se diferencia el marco de la Enseñanza para la Comprensión de un enfoque tradicional? Ruta Maestra, 9, 26-32.

Bautista, A. (2017). La foto-elicitación en la formación permanente de maestros de educación primaria. Alteridad, 12 (2), 1-16. doi: http://dx.doi. org/10.17163/alt.v12n2.2017.06

Belén, C. (2019). En busca de una docencia transformada: ¿cómo renovar culturas institucionales si no alteramos la formación de los docentes y sus prácticas? Revista de currículum y formación del profesorado, 23 (2), 232-258).

Bolívar, R. M. (2019). Investigar la práctica pedagógica en la formación inicial de maestros. Pedagogía y Saberes, 51, 9-22. 
Buitrago, R. (2018). Cotidianidad y retos para el profesorado. Práxis \& Saber, 9 (19), 9-18. https:// doi.org/10.19053/22160159.v9.n19.2018.7919

Canabal, C., \& Margalef, L. (2017). La retroalimentación: la clave para una evaluación orientada al aprendizaje. Revista de Currículum y Formación de Profesorado, 21 (2), 149-170.

Castro, M. C. (2019). Ambientes de aprendizaje. Sophia, 15 (2), 40-54.

Cerrón, W. (2019). La investigación cualitativa en educación. Horizonte de la Ciencia, 9 (17), 1-8.

Cifuentes, J. E. (2015). Enseñanza para la comprensión: opción para mejorar la educación. Revista Educación y Desarrollo social, 9 (1), 70-80.

Cifuentes, J. E. (2018). Movimiento en el aprendizaje de las rutinas de pensamiento en profesores. Praxis \& Saber, 9 (19), 121-139. https://doi. org/10.19053/22160159.v9.n19.2018.7924

Cifuentes, J. E. (, 2019). Aprendizaje del marco de la enseñanza para la comprensión en profesores: un abordaje desde las trayectorias de pensamiento. Revista Virtual Universidad Católica del Norte, 57, 3-23. https://doi.org/10.35575/rvucn. n57a2

Chaves, M., \& Barrios, L. (2017). La investigación acción y el aprendizaje por proyectos en el marco del modelo pedagógico Enseñanza para la comprensión. Experiencia del colegio Visión Mundial en comunidades vulnerables de Montería. Panorama, 11 (21), 29-51.

Ghitis, T. (2012). Trayectorias de pensamiento en la construcción de conceptos pedagógicos en estudiantes de Maestría en Pedagogía (Tesis de Maestría). Universidad de La Sabana. Chía, Colombia.

Hernández, R., Fernández, C., \& Baptista, P. (2014). Metodología de la investigación. México: McGraw Hill.

Libedinsky, M. (2016) ¿Cómo enseñar para la transferencia en aulas en línea de nivel superior? SIGNOS EAD, 1-14.
Mosquera, A. M. (2019). Tendencias investigativas en educación en Colombia: revisión documental. Sophia, 15 (1), 1-4.

Nicastro, S., \& Greco, M. (2012). Entre trayectorias. Escenas y pensamientos en espacios de formación. Rosario, Argentina: Homo Sapiens Ediciones.

Perkins, D. (2014). Enseñar para la comprensión en el siglo XXI. Perkins et al. (Coords.), Enseñar para comprender Il:experiencias y propuestas para la Educación Superior 14-25. Argentina: Eduvim.

Ravanal, E. (2019). Descriptores e indicadores de una práctica de enseñanza efectiva según profesores de biología en servicio. Tecné, Episteme y Didaxis, 46, 123-137.

Restrepo, B. (2006). La Investigación-Acción Pedagógica, variante de la Investigación-Acción Educativa que se viene validando en Colombia. Revista de la Universidad de La Salle, 42, 92-101.

Ritchhart, R., Church, M., \& Morrison, K. (2014). Hacer visible el pensamiento. Buenos Aires: Paidós.

Romero, A., \& Sanz, T. (2017). ¿Tesis o examen de grado? Un dilema para la formación investigativa. ALTERIDAD, 12 (2), 1-14. https://doi.org/10.17163/ alt.v12n2.2017.09

Sánchez, L., \& Puerta, C. (2011). Aproximación conceptual al proceso de realimentación en la educación virtual. Revista Virtual Universidad Católica del Norte, 34, 8-34.

Stone, W. (1999). La enseñanza para la comprensión. Vinculación entre la investigación y la práctica. Buenos Aires, Argentina: Paidós.

Tovar, E. (2019). Implementación de estrategias pedagógicas constructivistas mediadas por las herramientas Web 2.0 para el fortalecimiento de la comprensión teórica en los contenidos conceptuales de las ciencias naturales y la educación ambiental. Revista Interamericana de Investigación, Educación y Pedagogía, 12 (2), 71-112.

Unesco, (2017). Resumen del informe de seguimiento de la educación en el mundo. Rendir cuentas en el ámbito de la educación: cumplir nuestros compromisos. París, Francia. 
Vega, M., \& Appelgren, D. (2019). E-portafolio: una herramienta para el desarrollo de la práctica reflexiva de profesores en formación. Praxis, 15 (1), 57-68. http://dx.doi.org/10.21676/23897856.2983

Villa, E. (2019). La sistematización de experiencias, una estrategia de la investigación anti-hegemónica. El Ágora USB, 19 (2), 547-557. https://doi. org/10.21500/16578031.4389

Villamizar, E., Montero, M., González, M., \& Castillo, M. (2019). Mejorando los niveles de comprensión en Química a partir del enfoque de la enseñanza para la comprensión. Conocimiento Educativo, 6, 37-51.

Vives, T., \& Varela, M. (2013). Realimentación efectiva. Investigación en Educación Médica, 2 (6), 112-114.

Wilson, D. (2002). La retroalimentación a través de la Pirámide y la Escalera de Retroalimentación. "Seminario: Cerrando la brecha: I Encuentro de tutores latinoamericanos en línea", Miami. 